\title{
Inspiration comes naturally
}

\section{A collection of articles in this issue focuses on attempts to mimic aspects of natural-product biosynthesis for the identification of new drugs.}

Natural products and their derivatives have long been, and remain, a significant source of pharmaceuticals ${ }^{1}$. Aspirin (acetylsalicylic acid) has been sold as a drug since 1899 , but the pain-relieving effects of plant extracts now known to contain salicylic acid have been appreciated since antiquity. Similarly, the antimalarial properties of the bark of the cinchona tree were recognized long before the use of quinine. More recently, paclitaxel (Taxol) - an anticancer drug - was isolated in 1967 from the bark of the pacific yew tree. Coming right up to date, the breast cancer drug eribulin (Halaven) - approved by the US Food and Drug Administration in 2010 - is a simplified, albeit still rather complex ${ }^{2}$, synthetic analogue of halichondrin $\mathrm{B}$, which is produced by the marine sponge Halichondria okadai.

As noted in an earlier Editorial ${ }^{3}$, however, the prevalence of drugs derived from natural products is "more to do with nature's ability to produce such an enormous and diverse range of structures than any grand plan to provide a cure for each and every disease". Two Articles in this issue attempt to tap into this ability, taking inspiration from different aspects of natural-product biosynthesis to identify new bioactive molecules.

The dizzying array of natural-product structures is ultimately derived from a small number of building blocks, which can be arranged in various permutations. It is this aspect of natural-product biosynthesis that captured the attention of Yi-Lin Huang and Jeffrey Bode, and inspired their development of what they dub 'synthetic fermentation' (page 877). The process uses the groups' previously developed ketoacid-hydroxylamine (KAHA) ligation ${ }^{4}$ to stitch together 23 building blocks into a library of 6,000 unnatural peptides. Screening of the library and deconvolution of the results led to identification of a hepatitis $C$ virus protease inhibitor.

George Karageorgis, Stuart Warriner and Adam Nelson take a different approach (page 872) - in which they design a substrate with multiple possible reactivities and are deliberately unaware of the reaction outcome - allowing the biological activity to steer them towards a product and, at the same time, a way to make it. The method is intended to mimic the way in which organisms that produce natural products with a functional benefit develop a survival advantage and thus pass on to descendants the ability to make the compound. As a proof of concept, they embed a molecular fragment found in androgen receptor ligands within a reaction substrate and subject it to multiple different reaction conditions. Products that show desirable activity are identified and the route to them optimized in further rounds of screening. They use the process to identify a new androgen receptor antagonist.

\section{The dizzying array of natural-product structures is ultimately derived from a small number of building blocks, which can be arranged in various permutations.}

In an accompanying News and Views article (page 851), Derek Lowe describes how these approaches are essentially a modern take on combinatorial chemistry, but - to soothe the concerns of any battlescarred medicinal chemists who may, as a result, eye them with suspicion - he highlights two important aspects that might make these approaches more successful. Both methods require minimal purification of products before testing and - though they rely on the screening of a mixture they describe how an active component can be rapidly identified. He also muses on the possibility of a "merged version of the two processes: aqueous, no-workup chemistry, using discrete compounds in a user-defined order, which could also be sent down different synthetic pathways by varying the reaction conditions". Coincidentally, Lowe also notes how these developments in screening for biological activity dovetail with recent developments in screening for reaction discovery. Collins, Gensch and Glorius provide an overview of the opportunities and challenges in this exciting area of contemporary synthesis in a Review Article also in this issue (page 859).
The corresponding authors of the two research Articles also answered some questions about the origins and future directions of their studies (Interviews on pages 845 and 846). As Warriner explains, activity-directed synthesis is different from the usual process of developing structure-activity relationships because "the structures of products are only ever elucidated after biological function has been demonstrated". Nelson points out that while the reaction substrate used in their study incorporates a molecular fragment found in known drugs, the active products identified "were unlikely to have ever been explored in a conventional library synthesis".

Asked to expand on the similarities and differences between synthetic fermentation and previous library syntheses, Jeffrey Bode explains that he sees the work "as an implementation of Sharpless's vision for click chemistry" - diverse function from a few good reactions ${ }^{5}$. He goes on to suggest that the ease with which the libraries are generated and the fact that the chemistry requires no special training could even make it suitable for a 'citizenscience' approach to drug discovery. While acknowledging that there will always be concerns about safety and security when discussing the idea of laboratories in the home, he suggests that opening up the science in this way might help to alleviate the oft-lamented public image problem of chemistry.

The structures and inherent diversity of natural-product structures have long inspired efforts to create 'natural productlike' libraries. The methods described in this issue aim to exploit not just the final structure of natural products, but also the processes by which those compounds are created. With continued development, the hope will be that these approaches can help to maintain the legacy of natural products in drug discovery.

\footnotetext{
References

1. Newman, D. J. \& Cragg, G. M. J. Nat. Prod. 70, 461-477 (2007).

2. Yu, M. J., Zheng, W. \& Seletsky, B. M. Nat. Prod. Rep. 30, 1158-1164 (2013).

3. Nature Chem. 4, 955 (2012).

4. Bode, J. W., Fox, R. M. \& Baucom, K. D. Angew. Chem. Int. Ed. 45, 1248-1252 (2006).

5. Kolb, H. C., Finn, M. G. \& Sharpless, K. B. Angew. Chem. Int. Ed. 40, 2004-2021 (2001).
} 